\title{
Velocity of centre of pressure as a predictor of walking speed
}

\author{
Noël LW Keijsers ${ }^{1 *}$, Niki M Stolwijk1, Jan-Willem K Louwerens², Jaak Duysens ${ }^{1,3}$ \\ From 3rd Congress of the International Foot and Ankle Biomechanics Community \\ Sydney, Australia. 11-13 April 2012
}

\section{Background}

Walking speed is one of the best measures of overall walking ability. In plantar pressure measurements, walking speed is usually assessed using a stopwatch, photocells, or camera systems. As a simple alternative, contact time can be used but contact time and walking speed are only moderately correlated. Because walking speeds alters foot loading, the centre of pressure might be of more value to indicate walking speed. Therefore, the purpose of this study is to assess walking speed using the velocity of the centre of pressure (VCOP).

\section{Materials and methods}

Thirty-three subjects (range 19-77 years) walked over a Foot Scan pressure plate (Rsscan Int) at three speed conditions; slow, preferred, and fast. Contact time and mean VCOP during stance were calculated for each subject and condition. Walking speed was measured by a Vicon motion analysis system using a marker on the heel. Linear regression analysis was used to indicate the relation between walking speed and the independent variables: contact time, mean VCOP during stance, and both combined for all walking conditions separately and together. Finally, stance phase was divided in 5 equal time frames; the mean VCOP was calculated for each frame and used as 5 independent variables (5 VCOP)

\section{Results}

When all walking speeds were combined, walking speed was highly correlated with mean VCOP and contact time (see Table 1). However, correlation coefficients values were much lower for the preferred, low, and fast walking speed separately. Mean VCOP showed larger correlation coefficients than contact time. Adding
Table 1 Correlation coefficient values ( $r$ )

\begin{tabular}{lllll}
\hline Walking speed & Mean VCOP & Contact time & Both & 5 VCOP \\
\hline Slow & 0.80 & -0.78 & 0.82 & 0.84 \\
Preferred & 0.90 & -0.69 & 0.90 & 0.91 \\
Fast & 0.60 & -0.45 & 0.61 & 0.76 \\
All walking speeds & 0.94 & -0.88 & 0.94 & 0.96 \\
\hline
\end{tabular}

contact time, the correlation coefficient increased minimally. Walking speed was best predicted when the 5 VCOP values were used.

\section{Conclusions}

Mean VCOP is a better predictor for walking speed than contact time. Using more detailed information of the VCOP, the prediction of walking speed can be further increased.

\section{Author details \\ 'Department of RD\&E, Sint Maartenskliniek, Nijmegen, the Netherlands. ${ }^{2}$ Orthopaedics, Sint Maartenskliniek, Nijmegen, the Netherlands. ${ }^{3}$ Research Center for Movement Control and Neuroplasticity, Department of Biomedical Kinesiology, Katholieke Universiteit Leuven, Leuven, Belgium.}

Published: 10 April 2012

doi:10.1186/1757-1146-5-S1-028

Cite this article as: Keijsers et al:: Velocity of centre of pressure as a predictor of walking speed. Journal of Foot and Ankle Research 20125 (Suppl 1):O28.

\footnotetext{
* Correspondence: n.keijsers@maartenskliniek.nl

'Department of RD\&E, Sint Maartenskliniek, Nijmegen, the Netherlands

Full list of author information is available at the end of the article
} 